\title{
A prática da humanização da relação médico-paciente nos alunos de primeiro período de medicina da Universidade Severino Sombra: a visão do calouro que se tornou monitor- Um relato de experiência
}

The practice of humanization of the doctor-patient relationship in the first-degree medical students of the Severino SombraUniversity. The freshman vision that became a monitor. An experience report

La práctica de la humanización de la relación médico-paciente en estudiantes de medicina de primer año en la Universidad Severino Sombra

Sara Cristine Marques ${ }^{1}$, Lívia Liberata Barbosa Bandeira ${ }^{2}$, Ivan Lucas Picone Borges dos Anjos ${ }^{3}$, Thais Lemos de Souza Macedo ${ }^{4}$, Dandhara Martins Rebello ${ }^{5}$ Eucir Rabello ${ }^{6}$, Ivana Picone Borges de Aragão ${ }^{* *}$

Como citar esse artigo. Marques, SC; Bandeira, LLB; dos Anjos, ILPB; Macedo, TLS; Rebello, DM; Rabello, E; de Aragão, IPB . A prática da humanização da relação médicopaciente nos alunos de primeiro período de medicina da Universidade Severino Sombra: a visão do calouro que se tornou monitor- Um relato de experiência. 2019 Jul./Dez.; 10 (2): 28-31.

\section{Resumo}

A Prática da Humanização da Relação Médico-Paciente nos Alunos de Primeiro Período de Medicina da Universidade Severino Sombra. Projeto Calouro Humanoé um projeto tanto da Pró-Reitoria de Pesquisa e Pós-Graduação, como da Pró-Reitoria de Extensão Universitária da Universidade de Vassourasvoltado para os alunos do primeiro período do curso de medicina de cada semestre letivo - o calouro humano. Possui duração de 14 semanas envolvendo também, monitores, que são alunos de diversos períodos, exceto o primeiro, além dos professores da disciplina de Programa de Aproximação a Prática Médica 1. Ele consiste em trabalhar com o aprendizado e a utilização dos princípios da humanização da relação médico-paciente e inserir o aluno desde o princípio da faculdade no ambiente hospitalar visando o paciente como pessoa portadora de uma doença. Quando perguntados a respeito da experiência ao executarem as suas funções, cerca de $80 \%$ dos alunos responderam positivamente em relação a vivência nessa atividade. Trata-se de um projeto de importância para a construção gradual do desenvolvimento da habilidade de empatia e comunicação dos alunos envolvidos em ambas as funções.

Palavras-chave: Humanização da Assistência, Estudantes de Medicina, Educação Médica.

\begin{abstract}
The Practice of Humanization of the Doctor-Patient Relationship in First-Year Students at Severino Sombra University. Human Freshman Project is a project of both the Research and Post-Graduation Department and the Dean of University Extension of the University of Vassouras, aimed at students of the first period of the medical course of each semester - the human freshman. It has a duration of 14 weeks also involving monitors, who are students from different periods, except the first one, besides the teachers of the Program Approach to Medical Practice 1. It consists of working with the principles of humanization of the doctor-patient relationship and insert the student from the beginning of college in the hospital environment aiming at the patient as a person with a disease. When asked about their experience in performing their duties, about $80 \%$ of the students answered positively regarding their experience in this activity. It is a project of importance for the gradual construction of the development of empathy and communication skills of the students involved in both functions.
\end{abstract}

Keywords: Humanization of Assistance, Students Medical, Education Medical.

Afiliação dos autores: 1 Acadêmico do Curso de Medicina, IC projeto de pesquisa e extensão, Universidade de Vassouras. RJ, Brasil. Email: saracmarques@icloud.com ORCID https://orcid.org/0000-0002-8205-8112

2 Acadêmica do Curso de Medicina, IC projeto de pesquisa e extensão, Universidade de Vassouras. RJ, Brasil. Email: livialibertb@gmail.com ORCID: https://orcid.org/0000-0002$7305-3504$

3 Acadêmico do Curso de Medicina, IC projeto de pesquisa e extensão, bolsista PIBIC CNPq,Universidade de Vassouras. RJ, Brasil. Email: ilpba@hotmail.com ORCID: https:// orcid.org/0000-0003-4211-1887

4 Acadêmico do Curso de Medicina, IC projeto de pesquisa e extensão, Universidade de Vassouras. RJ, Brasil. Email: thais.lsm@hotmail.com ORCID: https://orcid.org/0000-00024461-7470

5. Acadêmico do Curso de Medicina, IC projeto de pesquisa e extensão, Universidade de Vassouras. RJ, Brasil. Email: dandhararebello@gmail.com ORCID: https://orcid.org/00000002-9250-9378

6. Coordenador do projeto de extensão, do Programa de Aproximação a Prática Médica 1 e e coordenador do curso de Medicina daUniversidade de Vassouras. RJ, Brasil. Email: eucir.rlk@terra.com ORCID: https://orcid.org/0000-0002-9501-0353

7. Coordenadora do projeto de pesquisa e docente do Curso de Medicina do Programa de Aproximação a Prática Médica 1 da Universidade de Vassouras. RJ, Brasil. Email: ivanapicone@globo.com ORCID: https://orcid.org/0000-0002-4295-0165

* Email de correspondencia: ivanapicone@globo.com

Recebido em: 07/09/19. Aceito em: 09/12/19. 


\section{Resumen}

La práctica de la humanización de la relación médico-paciente en estudiantes de primer año de la Universidad Severino Sombra La humanización de estudiantes de primer año es un proyecto del Departamento de Investigación y Postgrado y del Decano de Extensión Universitaria de la Universidad de Vassouras, dirigido a estudiantes del primer periodo del curso de medicina de cada semestre: el estudiante de primer año humano. Tiene una duración de 14 semanas que también involucra a monitores, que son estudiantes de diferentes períodos, excepto el primero, además de los maestros del programa de acercamiento a la practica medica 1 (Semiología). Consiste en trabajar con el aprendizaje y el uso de los principios de humanización de la relación médico-paciente e inserte al estudiante desde el comienzo de la universidad en el entorno hospitalario con el objetivo de que el paciente sea una persona con una enfermedad. Cuando se les preguntó sobre su experiencia en el desempeño de sus tareas, aproximadamente el $80 \%$ de los estudiantes respondieron positivamente sobre su experiencia en esta actividad. Es un proyecto de importancia para la construcción gradual del desarrollo de las habilidades de empatía y comunicación de los estudiantes involucrados en ambas funciones.

Palabras clave: Humanización de la Atención, Estudiantes de Medicina, Educación Médica.

\section{Introdução}

Aentrada na faculdade de medicina é um momento de grande expectativa pelo ingressante quando chega em um ambiente completamente novo e ainda desconhecido, repleto de expectativas sobre o futuro.Atualmente, muitas escolas médicas praticam a inserção mais precoce desse estudante no ambiente hospitalar, onde buscam que esse estudante seja gradativamente treinado para lidar com os pacientes, buscando compreendê-los como pessoas, seres humanos, o que vai muito além da doença que possui e sim um indivíduo com sentimentos, preocupações e expectativas.

De acordo com Edson Dell Amore', os estudantes de medicina irão experimentar mudanças comportamentais ao longo do curso, essa sendo desencadeada pela convivência com o adoecimento, o sofrimento e a dificuldade para prover cuidados trazendo como efeito colateral o afastamento do estudante, como um mecanismo de defesa, consequentemente na desumanização do cuidado médico.

\section{A humanização na medicina}

A prática da humanização da medicina e o surgimento do modelo biopsicossocial vem da necessidade de buscar uma visão mais holística do homem, de entender que os aspectos psicológicos estão ligados aos biológicos no processo de adoecimento e cuidado $^{2}$. Ela também pode ser vista como uma forma de ampliar a comunicação ${ }^{3}$. É muito importante que seja feita a incorporação da humanização por todos os envolvidos no processo de produção de saúde, é necessário que haja acolhimento, solidariedade e compaixão para que possa ser feita a construção da relação entre o usuário e o que provém o cuidado ${ }^{6}$. Há então a necessidade de mudança, sendo necessário resgatar a imagem do paciente como único e respeitá-lo dessa forma para que seja possível tratar sua doença ${ }^{7}$.

A medicina atual precisa parar de enxergar o paciente apenas como uma doença a ser tratada, é necessário vê-lo de uma forma mais humanitária, para que consiga obter uma interação de individualidade com aquele que busca o atendimento e para que seja realizado o acolhimento. Um médico competente nasce quando um agente que deseja ser um transformador social escolhe a carreira médica ${ }^{8}$.

\section{A chegada do aluno na faculdade}

O aluno ao iniciar sua vida acadêmica, ao enfrentar as primeiras idas ao hospital e o primeiro contato com o paciente, se sente inseguro e sem saber como lidar, conversar e interagir, pois, para ele é algo novo. A importância desse projeto é de acolher esse aluno e orientá-lo nessa tarefa, isso através da prática, onde o aluno e o monitor, juntamente com o professor, compartilham conhecimento e experiências.

\section{A relação médico-paciente}

A construção da relação médico-paciente é capaz de causar ansiedade para o doente, sobretudo aqueles de classes mais populares, geradas pela dificuldade de comunicação e pela sensação desses pacientes de que o médico não é franco com eles ${ }^{4} a l e ́ m$ sensação de inferioridade que alguns pacientes sentem diante do médico, o qual muitas vezes se apresenta numa postura distante, na população de baixa renda.Acredita-se que a divisão da medicina em diversas especialidades também possa ter contribuído com o enfraquecimento dessa relação entre médico e paciente, necessitando então da disseminação da humanização na área de saúde, de forma a restaurar o foco do cuidado ao doente não ser limitado somente a doença, mas na pessoa como um todo 5 .

Um dos pontos positivos do projeto é justamente trabalhar o desenvolvimento de sua relação com o paciente desde a sua entrada na universidade, trazendo uma visão mais humanizada do processo saúde doença e também para que aqueles alunos recém chegados, que em um primeiro momento se sentem inseguros 
na abordagem do paciente, possam desenvolver essa habilidade com o auxílio de professores e alunos veteranos.

Objetivo: relatar a experiência de alunos de primeiro período de medicina no desenvolvimento da habilidade de empatia e comunicação voltados a utilização dos princípios da humanização.

\section{Metodologia}

Trata-se de um projeto de pesquisa, inserido na Pró-Reitoria de Pesquisa e Pós-graduação da Universidade de Vassouras, parecer do Conselho de Ética em Pesquisa (CEP) n ${ }^{\circ}$ 1.963.944, com aprovação no ano de 2016 e projeto de extensão junto a PróReitoria de Extensão Universitária da Universidade de Vassouras, com início em 2017.

Consiste em inserir o aluno do primeiro período de medicina (APPM) no ambiente hospitalar, acompanhado de monitores de períodos acima do deles, os quais já foram participantes como APPM, para duas visitas semanais as enfermarias do Hospital Universitário de Vassouras (HUV). A princípio, a ideia é que eles entrevistem o paciente de um mesmo leito hospitalar pré-estabelecido, acompanhado dos monitores, todas as semanas de projeto. Em caso de alta do paciente, é feita a troca para outro paciente que tiver internado naquele leito hospitalar. Esses pacientes,após assinatura de um Termo de consentimento livre e esclarecido (TCLE), irão responder um questionário anônimo, onde serão feitas perguntas relacionadas ao atendimento hospitalar, se ele julga receber um atendimento humanizado, a respeito da presença dos alunos de medicina nas enfermarias de forma constante, sobre suas necessidades e na possibilidade de ser ajudado pelo APPM. Cada grupo de alunos fica responsável por um leito, sendo supervisionados pelos monitores ou pelos professores. É pedido para que eles tentem desenvolver uma conversa com o paciente e dentro dessa conversa sejam feitas as perguntas dos questionários, tentando tornar assim um momento mais agradável para o paciente e possibilite maior desenvolvimento comunicativo do aluno.

Recentemente, dado ao sucesso e adesão, houve a inclusão desse projeto, na forma de projeto de extensão universitária, atuando fora do horário destinado ao cronograma letivo, à disciplina de Programa de Aproximação a Prática Médica 1 (PAPM1), fato de grande relevância pois ele complementa de forma prática,o conteúdo ensinado na PAPM1, como a relação médico-paciente, o processo saúde doença, a medicina centrada na pessoa e a entrevista com o paciente sobre sua doença e história de vida. Essa inclusão do projeto à PAPM1 tornou a participação dos APPM obrigatória, como parte do programa de ensino da PAPM1.

\section{Resultados}

\section{Minha experiência pessoal com o projeto}

Como "ex-caloura humana", durante o ano de 2018 e atualmente monitora desde o início de 2019 , foi possível perceber como é importante e necessário a existência de projetos como esse durante o curso de graduação médica. O aprendizado é progressivo e possibilita uma aproximação gradual aluno com o paciente, possibilitando assim com que aqueles alunos considerados mais tímidos possam ir desenvolvendo sua habilidade de comunicação. Voltar ao projeto como monitora possibilitou com que eu pudesse levar aos calouros do meu grupo os pontos positivos e experiências que meus monitores me proporcionaram e a corrigir aqueles pontos nos quais pudesse ter havido problemas. Isso tende a gerar uma reação em cadeia, onde o que é positivo vai sendo disseminado com o passar do tempo, a cada período, e o que onde há necessidade de ajustes vai sendo corrigido, contribuindo cada vez mais para a melhoria do projeto.

O trabalho desenvolvido pelo projeto tem impacto direto na construção da relação que esse APPM terá com os pacientes desde o início de sua vida acadêmica e no decorrer de sua vida profissional, sendo capaz de transformar essa prática em algo natural para sua carreira médica e também o capacitando a ser um disseminador desses princípios e ações.

\section{O progresso dos alunos novos}

Ao longo das 14 semanas de projeto foi possível acompanhar o progresso de cada calouro de nosso grupo de monitoria, onde cada grupo monitor fica responsável por cerca de 6 a 8 alunos, que podem ser divididos em duplas ou trios. Nas semanas iniciais há uma dependência muito maior da participação do monitor em todo processo de diálogo com o paciente, tanto na apresentação quanto na condução da aplicação do questionário. Com o passar do tempo, após algumas visitas, é possível notar a evolução dos calouros que da metade do projeto em diante (de um modo geral) conseguem administrar toda a visita com muita autonomia.

\section{Os monitores}

A seleção é feita de forma voluntária e atualmente, no segundo semestre de 2019, contamos com cerca de 60 monitores de variados períodos da faculdade de medicina, entre o $2^{\circ}$ e $8^{\circ}$ período. Promovendo assim também uma interação entre os estudantes.

Os alunos "calouros humanos" são orientados a 
responderem um questionário ao longo do projeto, onde é perguntado a opinião dele a respeito do projeto, qual o efeito percebido por ele em relação ao contato com o paciente e sobre a percepção dele se através daquela visita ele sentiu que pôde ajudar de alguma forma esse paciente. É válido salientar que esses questionários são anônimos, de forma a buscar obter uma resposta sincera por parte dos alunos.

Dos 648 questionários respondidos, entre os anos de 2017 até o primeiro semestre de 2019, 520 alunos classificaram a experiência com o projeto como ótima, 102 como boa, 20 como regular e 6 como ruim. Quanto a sensação de aproximação ao paciente, 611 relataram que houve aproximação com o paciente, 35 referiram não terem notado alterações, 1 não soube responder e apenas 1 nos relatou que houve afastamento do paciente. Relacionado ao apoio a necessidade do paciente, 501 informaram terem sido atendidas, 127 atenderam parcialmente, 13 não atenderam e 7 não informaram.Em um dos questionários, algum dos alunos escreveu que o projeto o ajudou a enfrentar a timidez na abordagem do paciente.

\section{Conclusão}

Trata-se de um projeto de grande importância para o desenvolvimento do aluno do primeiro período de medicina que ainda está em processo de adaptação com as mudanças que a entrada na faculdade provoca. É uma forma de mostrar na prática como é feita a construção da relação médico-paciente, de que o paciente é muito mais do que uma doença, do que o quadro clínico que apresenta. Ele tem uma história, sentimentos e necessidades. Isso também é válido para os monitores que continuarão trabalhando no desenvolvimento de sua capacidade de comunicação e reforçando cada vez mais os benefícios do atendimento humanizado e o bem que isso pode trazer ao paciente.

\section{Referências}

1 Amore Filho, Edson Dell, Dias, Ruth Borges, \& Toledo Jr, Antônio Carlos de Castro. (2018). Ações para a Retomada do Ensino da Humanização nas Escolas de Medicina. Revista Brasileira de Educação Médica, 42(4), 14-28. https://dx.doi.org/10.1590/1981-52712015v42n4rb20180056

2. Alves, Antonia Núbia de Oliveira, Moreira, Simone da Nóbrega Tomaz, Azevedo, George Dantas de, Rocha, Vera Maria da, \& Vilar, Maria José. (2009). A humanização e a formação médica na perspectiva dos estudantes de medicina da UFRN - Natal - RN - Brasil. Revista Brasileira de Educação Médica, 33(4), 555-561. https://dx.doi.org/10.1590/S010055022009000400006

3. Rios, Izabel Cristina, \& Sirino, Caroline Braga. (2015). A Humanização no Ensino de Graduação em Medicina: o Olhar dos Estudantes. Revista Brasileira de Educação Médica, 39(3), 401-409. https://dx.doi.org/10.1590/1981$52712015 \mathrm{v} 39 \mathrm{n} 3 \mathrm{e} 00092015$

4.https://www.s cielosp.org/s cielo.php? pid=S 0102 $311 X 1999000300023 \&$ script=sci arttext\&tlng=en\#ModalArticles
5. Rabello, E; Bandeira, LLB; dos Anjos, ILPB; dos Santos, CT; Macêdo, TLS; da Silva, CPO; Rebello, DM; Dantas, PRS; Soares, RR; Neto, ARB; de ARagão, IPB. Experiência inicial com a prática de humanização na relação médicopaciente em alunos do primeiro período do Curso de Medicina da Universidade de Vassouras . Revista Fluminense de Extensão Universitária 2019 Jan/Jun.; 09 (1): 09-13

6. EGO S.;GOMES A.P.; BATISTA R. S.; Bioética e Humanização como Temas Transversais na Formação Médica. Revista brasileira de educação médica; 32 (4) : 482 - $491 ; 2008$

7. Cristina, Muccioli, Campos, Mauro S. Q., Dantas, Paulo E.C., Goldchmit, Mauro, Bechara, Samir J., Costa, Vital Paulino, \& Matayoshi, Suzana. (2007). A humanização da medicina. Arquivos Brasileiros de Oftalmologia, $70(6), 897$

8. Silveira, ALP. Rede Humaniza SUS [internet]. [atualizado em 26 ago 2016; citado em 05 set 2019] Pela Humanização da Medicina. Disponível em: http://redehumanizasus.net/95192-pela-humanizacao-da-medicina/ 